\title{
Diagnostic rapide des mycoses opportunistes par la détection d'antigènes circulants
}

Le diagnostic des mycoses opportunistes à Candida et Aspergillus est souvent difficile. L'amélioration des techniques de dosage des antigènes solubles dans le sang ou dans $d$ 'autres fluides biologiques permet d'envisager de prochains progrès.

\section{Louis de Repentigny \\ Professeur adjoint de clinique. Département de microbiologie et d'immunologie. Université de Montréal.}

\section{Michèle Boushira Chargée de recherche. Département de microbiologie et d'immunologie. Université de Montréal.}

\section{RÉFÉRENCES \\ 1. Fraser DW, Ward JI, Ajello L, Plikaytis BD. Aspergillosis and other systemic mycoses : the growing problem. JAMA $1979 ; 242$ : 1631-5.}

2. Rinaldi MG. Invasive aspergillosis. Rev Infect Dis 1983 ; 5 : 1061-77.

3. Horn R, Wong B, Kiehn TE, Armstrong D. Fungemia in a cancer hospital : changing frequency, earlier onset, and results of therapy. Rev Infect Dis $1985 ; 7$ : 646-55.

\section{ADRESSE}

L. de Repentigny, M. Boushira : département de microbiologie et d'immunologie, faculté de médecine, université de Montréal, et centre de recherche pédiatrique, hôpital Sainte-Justine, es infections à champignons opportunistes sont devenues, au cours des vingt dernières années, une cause majeure de morbidité et de mortalité, en particulier chez les individus immunodéprimés. De 1970 à 1976, la mortalité causée par les deux infections à champignons opportunistes les plus fréquentes, la candidose et l'aspergillose invasives, a augmenté de $73 \%$ et de $127 \%$ respectivement [1]. L'augmentation du nombre total des cas rapportés a été attribuée à l'existence d'un plus grand nombre de sujets à risque, tels les patients atteints de cancer et les traitements qui en découlent, et à des méthodes diagnostiques plus agressives.

La candidose et l'aspergillose invasives sont particulièrement fréquentes chez les patients atteints de leucémie aiguê ou ayant subi une transplantation de moelle osseuse $[2,3]$; mais on retrouve également ces infections associées aux leucémies chroniques, aux lymphomes et aux tumeurs solides. La neutropénie constitue un risque majeur puisque les monocytes et les polynucléaires jouent un rôle primordial dans la défense contre les espèces Candida et Aspergillus [4, 5]. On retrouve également ces infections chez les enfants atteints de granulomatose septique dont les granulocytes, bien qu'en quantité normale, sont incapables de tuer le microorganisme ingéré parce qu'ils ne peuvent plus produire en quantité suffisante le peroxyde d'hydrogène et d'autres agents oxydants. Les transplantés rénaux sont également exposés parce que leurs macrophages sont incapables de détruire les conidies ingérées en raison de la corticothérapie [6]. Un dernier groupe à risque est constitué des patients sous hyperalimentation parentérale, en raison des risques septiques inhérents à cette procédure et, probablement, de l'immunosuppression qui accompagne la dénutrition. Le diagnostic de la candidose et de l'aspergillose invasives repose sur la détection des formes levures et pseudomycéliennes de Candida, ou de filaments mycéliens d'Aspergillus, dans les coupes histopathologiques ; le diagnostic est de préférence confirmé par la culture du champignon.

\section{La candidose}

L'autopsie des victimes de leucémies aiguës révèle une candidose invasive ou disséminée chez $15-30 \%$ des cas étudiés [7]. On pense généralement que la plupart des infections à Candida sont d'origine endogène puisque cette levure colonise le côlon de 20 à $50 \%$ des individus sains. La chimiothérapie antinéoplasique et l'utilisation prolongée d'antibiotiques peuvent respectivement 
endommager les microvillosités intestinales et favoriser la prolifération de Candida dans la lumière gastro-intestinale. Ces deux facteurs pourraient faciliter la migration de la levure à travers la paroi intestinale par un processus encore mal connu et appelé "persorption " [8]. Une fois persorbé, le Candida peut ensuite se propager à travers l'organisme et provoquer l'infection des organes profonds chez l'hôte immunodéprimé. Bien qu'il soit généralement admis que la candidose invasive est d'origine endogène, certaines méthodes de typage [9-11] permettent d'envisager l'étude de la transmission de la levure entre les individus à l'intérieur d'un hôpital. On peut identifier ainsi les souches en cause. Candida tropicalis a supplanté Candida albicans en tant que principal agent responsable des candidoses invasives. On retrouve fréquemment Candida parapsilosis dans les hémocultures de patients soumis à une hyperalimentation parentérale.

\section{L'aspergillose}

Une aspergillose invasive est diagnostiquée chez environ 12 à $15 \%$ des individus atteints de leucémie aiguë et représente, par sa fréquence, la deuxième infection à champignon opportuniste. L'épidémiologie de l'aspergillose invasive est différente de celle de la candidose invasive, puisque la maladie est généralement déclenchée par l'inhalation de conidies. Aspergillus est répandu dans le sol, l'eau, les végétaux en décomposition et les déchets organiques. $\mathrm{La}$ concentration des conidies dans l'air est un facteur prédisposant important : plusieurs épidémies d'aspergillose invasive sont apparues, chez des patients à risque, après des travaux effectués dans les faux plafonds ou les conduites d'air des hôpitaux. Les conidies d'Aspergillus inhalées se heurtent à deux lignes de défense [12]. La première, contre les conidies, est constituée par les macrophages alvéolaires. La seconde, formée par les granulocytes, protège contre la forme mycé- lienne. Les corticostéroïdes inhibent la capacité des macrophages de tuer les conidies. L'immunité naturelle à l'égard d'Aspergillus s'effondre seulement lors d'une atteinte combinée du système réticuloendothélial et des granulocytes ; dans ces conditions, l'Aspergillus peut se disséminer à travers tout l'organisme. Aspergillus fumigatus et Aspergillus flavus sont les espèces les plus fréquemment retrouvées dans l'aspergillose invasive.

\section{Diagnostic des mycoses}

Le diagnostic des mycoses graves demeure problématique. Seuls 15 à $40 \%$ des cas de candidoses invasives sont diagnostiqués à temps pour qu'on puisse entreprendre un traitement. La culture des sites superficiels permettrait de prévoir une candidose ou une aspergillose invasive, mais cette procédure est peu sensible et les résultats arrivent souvent trop tard pour influencer les décisions cliniques. Les hémocultures sont négatives dans plus de $50 \%$ des cas de candidose disséminée prouvés à l'autopsie et sont presque toujours négatives dans l'aspergillose invasive. Une autre voie de diagnostic serait donc bienvenue. Des tests immunologiques détectant les anticorps circulants antiCandida ou anti-Aspergillus [7] sont utilisés depuis de nombreuses années. Malheureusement, ces tests s'avèrent peu satisfaisants lorsqu'il s'agit de patients immunodéprimés, les plus susceptibles aux mycoses invasives. La faible sensibilité de ces tests peut être due à l'incapacité de l'hôte à produire des anticorps ou des quantités détectables d'agglutinines ou de précipitines. De plus, ils ne permettent pas de faire la distinction entre la colonisation et l'invasion profonde des tissus et sont donc peu spécifiques. Des tentatives ont été faites pour identifier et purifier des antigènes plus spécifiques et pouvant être utilisés pour la recherche de ces anticorps [7]. Récemment, un test immunoblot ${ }^{*}$ a permis de démon- trer, dans la candidose invasive, la présence d'anticorps dirigés contre une protéine cytoplasmique de poids moléculaire 48000 [13, 14]. De plus, les patients produisant ces anticorps ont bénéficié d'une évolution plus favorable, suggérant que ces anticorps confèrent une protection contre la maladie. Cette voie semble prometteuse.

Cependant, pour les patients profondément immunodéprimés chez lesquels une infection ne conduirait pas à un niveau détectable d'anticorps, des stratégies différentes s'imposent, notamment la détection dans le sang des antigènes ou des métabolites de Candida et d'Aspergillus. Des polysaccharides comme le mannanne de Candida et le galactomannanne d'Aspergillus, principaux antigènes de la paroi de ces champignons, ont fait l'objet d'une attention particulière. Le dosage dans le sérum de métabolites monosaccharidiques comme l'arabinitol et le mannose a également été proposé pour le diagnostic de la candidose invasive. Nous discuterons brièvement des travaux entrepris dans ce domaine.

\section{Candida}

La mannanne est le principal constituant antigénique de la paroi cellulaire des espèces Candida. Il est formé d'une chaîne linéaire $\alpha(1,6)$ fortement substituée par des chaînes latérales d'oligomannosides liées en $\alpha(1,2)$ et $\alpha(1,3)$. La longueur des chaînes latérales, la localisation des liaisons 1,3 et les substitutions phosphodiesters déterminent la spécificité antigénique. La spécificité des sérotypes $\mathrm{A}$ et $\mathrm{B}$ de Candida albicans est conférée par le mannanne. La structure du mannanne de Candida albicans sérotype $\mathrm{A}$ a été étudiée par Suzuki et Fukazawa [15]. Ils ont proposé (figure 1) une structure arborisée comportant un tronc protéinique et des branches linéaires composées de deux à trois résidus de mannose liés $(1,6)$

\footnotetext{
- Immunoblot ou western blot : séparation électrophorétique des protéines, transfert sur filtre et révélation à l'aide d'anticorps appliqués sur le filtre.
} 


\section{RÉFÉRENCES}

4. Diamond RD, Haudenschild CC. Monocyte-mediated serum-independent damage to hyphal and pseudohyphal forms of Candida albicans in vitro. $J$ Clin Invest 1981; 67 : 173-82.

5. Diamond RD, Clark RA. Damage to Aspergillus fumigatus and Rhizopus oryzae hyphae by oxidative and nonoxidative microbicidal products of human neutrophils in vitro. Infect Immun 1982 ; 38 : 487-95.

6. Gustafson TL, Schaffner W, Lavely GB Stratton CW, Johnson HK, Hutcheson RH. Invasive aspergillosis in renal transplant recipients : correlation with corticosteroid therapy. $J$ Infect Dis 1983 ; $148: 230-8$.

7. De Repentigny L, Reiss E. Current trends in immunodiagnosis of candidiasis and aspergillosis. Rev Infect $D$ is 1984 ; 6 : 301-12.

8. Krause W, Matheis H, Wulf $\mathrm{K}$. Fungaemia and funguria after oral administration of Candida albicans. Lancet 1969 ; i : 598-9.

9. Roman MC, Sicilia MJL. Preliminary investigation of Candida albicans biovars. $J$ Clin Microbiol 1983 ; 18 : 430-1.

10. Polonelli L, Archibusacci C, Sestito M, Morace G. Killer system : a simple method for differentiating Candida albicans strains. $J$ Clin Microbiol 1983 ; 17 : 774-80.

11. Odds FC, Abbott AB, Stiller RL, Scholer HJ, Polak A, Stevens DA. Analysis of Candida albicans phenotypes from different geographical and anatomical sources. $J$ Clin Micro biol 1983 ; 18 : 849-57.

12. Schaffner A, Douglas H, Braude A. Selective protection against conidia by mononuclear and against mycelia by polymorphonuclear phagocytes in resistance to Aspergillus. $J$ Clin Invest $1982 ; 69$ : 617-31.

13. Strockbine NA, Largen MT, Zweibel SM, Buckley HR. Identification and molecular weight characterization of antigens from Candida albicans that are recognized by human sera. Infect Immun 1984; $43: 715-21$.

14. Matthews RC, Burnie JP, Tabaqchali S. Immunoblot analysis of the serological response in systemic candidosis. Lancet 1984; ii : 1415-8.

15. Suzuki M, Fukazawa Y. Immunochemical characterization of Candida albicans cell wall antigens : specific determinant of Candida albicans serotype A mannan. Microbiol Immunol

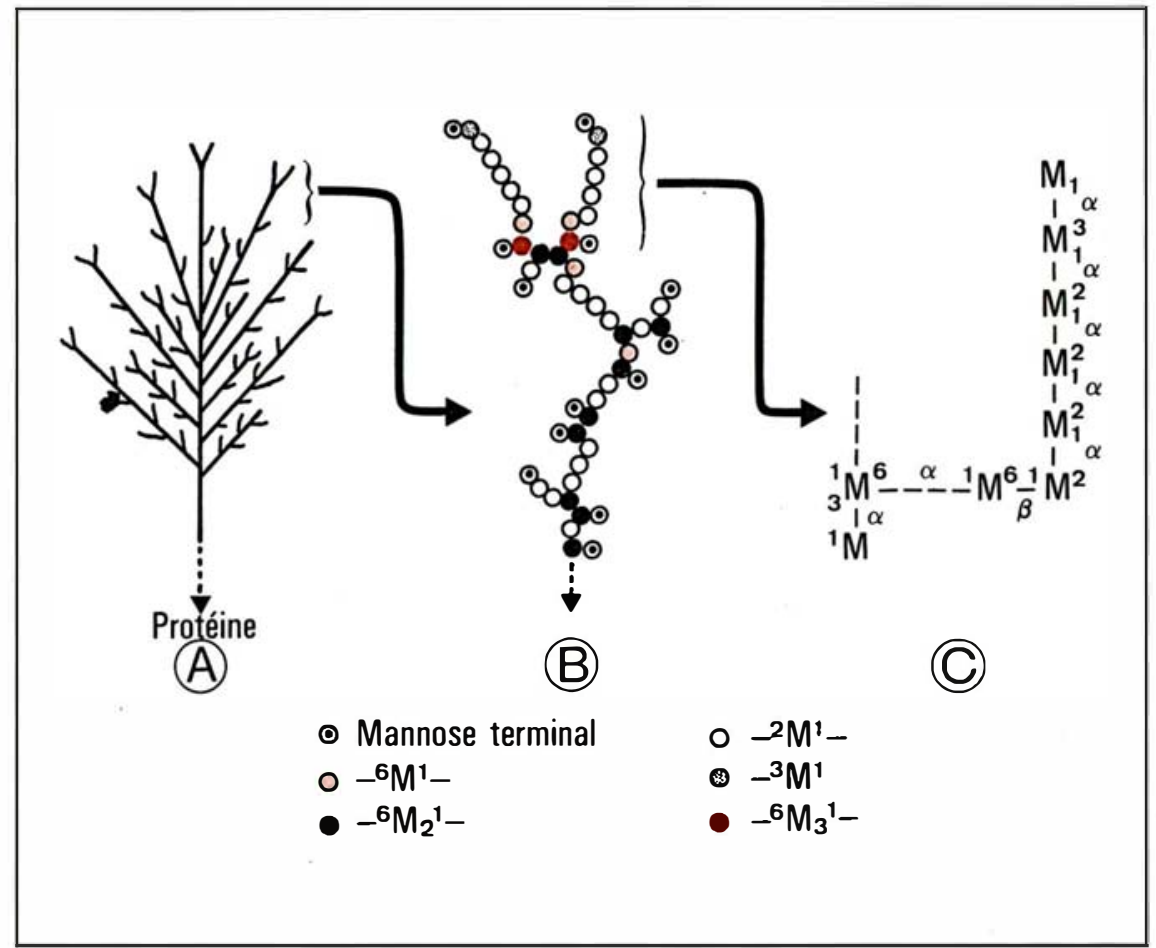

Figure 1. Modèlo de la structure du mannanno de Candida albicans sérotype A. (A) L'ensemble de la structure comporte un tronc protéinique et des branches linéaires composées de résidus de mannose. (B) Agrandissement d'une branche terminale contenant le facteur antigénique 6 spécifique au sérotype A. (C) Agrandissement du facteur antigénique 6. $M=$ mannopyranose. (Adapté de Suzuki et Fukazawa [15]).

alternant avec des résidus mannobiose liés $(1,2)$. La spécificité du stérotype A est localisée dans les petites branches terminales d'hexaose composées d'une liaison $\alpha(1,3)$ terminale en plus de quatre liaisons $\alpha(1,2)$. Le sérotype $\mathrm{B}$ est plus complexe et ne possède pas de liaison terminale 1, 3 mais plutôt un seul embranchement en $\mathrm{C}-1, \mathrm{C}-2$ ou $\mathrm{C}-3$ et une liaison interne 1,3 supplémentaire. Utilisant des antisérums spécifiques dirigés contre des facteurs antigéniques de Candida, il a été démontré que les sérotypes $\mathrm{A}$ et $\mathrm{B}$ possèdent respectivement les facteurs antigéniques 6 et $13 \mathrm{~b}$ et que le facteur 6 est un élément important pour l'identification du sérotype A. De plus, les techniques de précipitation quantitative ont pu faire la preuve que les mannannes de Candida tropicalis et de Candida albicans sont fortement apparentés.

La présence de mannanne dans le sérum a été rapportée pour la première fois par Axelsen et Kirk- patrick (1973) chez un patient atteint de candidose mucocutanée chronique. Depuis 1973, année où le mannanne sérique a été détecté par immunoélectrophorèse bidimensionnelle, des tests de plus en plus simples et sensibles ont été proposés : l'inhibition de l'hémaglutination, le test radioimmunologique ou les tests immunoenzymatiques. Deux facteurs compliquent la détection du mannanne sérique : en premier lieu, cet antigène circule à des concentrations inférieures à celles détectables par la plupart des tests sérologiques conventionnels (c'est-à-dire $\mathrm{ng} / \mathrm{ml}$ ); en second lieu, même chez les patients immunodéprimés, le mannanne circule sous la forme de complexes immuns qui doivent être préalablement dissociés. Au cours de récents travaux [7], la dissociation des complexes a été réalisée par brève ébullition du sérum dilué dans une solution de $\mathrm{Na}_{2}$ EDTA pour inhiber la coagulation. Le sérum dissocié est récupéré par centrifu- 
gation et réparti dans les puits de microplaques tapissés d'IgG de lapin anti-parois cellulaires de Candida albicans. Après incubation à $4^{\circ} \mathrm{C}$ et lavage, l'anticorps de détection marqué à la peroxydase permet de détecter des concentrations de mannanne de 1 à $100 \mathrm{ng} / \mathrm{ml}$. La réalisation de ce test immunoenzymatique se fait en trois heures (figure 2).

Notre étude réalisée avec cette méthode chez le lapin immunodéprimé et infecté expérimentalement par la voie intraveineuse avec Candida albicans a montré que la concentration de mannanne dans le sérum est maximale quatre jours après l'infection; elle diminue lors de l'apparition d'anticorps anti-mannanne [17]. Dans une étude rétrospective de patients leucémiques chez lesquels la présence ou l'absence d'une candidose invasive a été confirmée à l'autopsie, le test immunoenzymatique de type sandwich a donné une sensibilité de $65 \%$, une spécificité de $100 \%$, et une limite de détection de l'antigène de $1 \mathrm{ng} / \mathrm{ml}$ [18]. Plus récemment, des anticorps monoclonaux antimannanne ont été produits ; l'anticorps polyclonal de détection a été remplacé par une IgM monoclonale marquée à la peroxydase [19]. Ce test immunoenzymatique s'est révélé prometteur au cours d'études rétrospectives ; cependant, il devra être évalué à l'aide d'études multicentriques prospectives afin de confirmer son utilité en clinique.

Des antigènes autres que le mannanne ont été détectés dans le sérum de patients atteints de candidose invasive. Araj et al. ont découvert, dans le sérum de patients cancéreux [20], un antigène cytoplasmique de $C$. albicans qui semble être une protéine thermorésistante [20]. De plus, une trousse d'agglutination au latex qui est maintenant commercialement disponible permet de détecter dans le sérum un antigène qui est probablement une glycoprotéine de Candida. Mais cette méthode ne comporte pas la dissociation préalable des complexes immuns : voilà probablement $\mathrm{m} / \mathrm{s} n^{\circ} 2$ ool. 3, ftoriar 87 pourquoi, lors d'une évaluation clinique, l'antigène n'a été détecté que chez 5 des 18 patients atteints d'une candidose invasive [21].

Enfin, mise à part la recherche de mannarne sérique, le dosage de certains métabolites de Candida comme l'arabinitol et le mannose a été réalisé par chromatographie en phase gazeuse [7, 22]. En supposant que le mannose soit un métabolite du mannanne circulant in vivo, on a pensé qu'il pourrait servir de marqueur pour la candidose invasive. Cependant, nos études chez l'animal de laboratoire [17] et chez l'homme [18] ont montré que le dosage du mannose est trop peu sensible $(39 \%)$ et spécifique $(87 \%)$ pour être utilisé en clinique.

Une augmentation des concentrations sériques de $\mathrm{D}$-arabinitol chez les patients atteints de candidose invasive a été rapportée pour la première fois en 1979 [7]. Cette concentration était plus élevée chez les patients atteints d'insuffisance rénale que chez les patients ayant une fonction rénale normale. De plus, il a été démontré que l'arabinitol est produit in vitro par les espèces Candida. Cette substance est éliminée par le rein à la même vitesse que la créatinine et la concentration sérique d'arabinitol chez les insuffisants rénaux peut être ajustée en calculant le rapport arabinitol/créatinine. Il s'agit, semble-til, d'un marqueur quantitatif puisque la concentration sérique d'arabinitol est proportionnelle au décompte des colonies chez le rat atteint d'une candidose invasive expérimentale. La sensibilité et la spécificité du rapport arabinitol/créatinine varient largement selon les études; sa signification clinique demeure controversée.

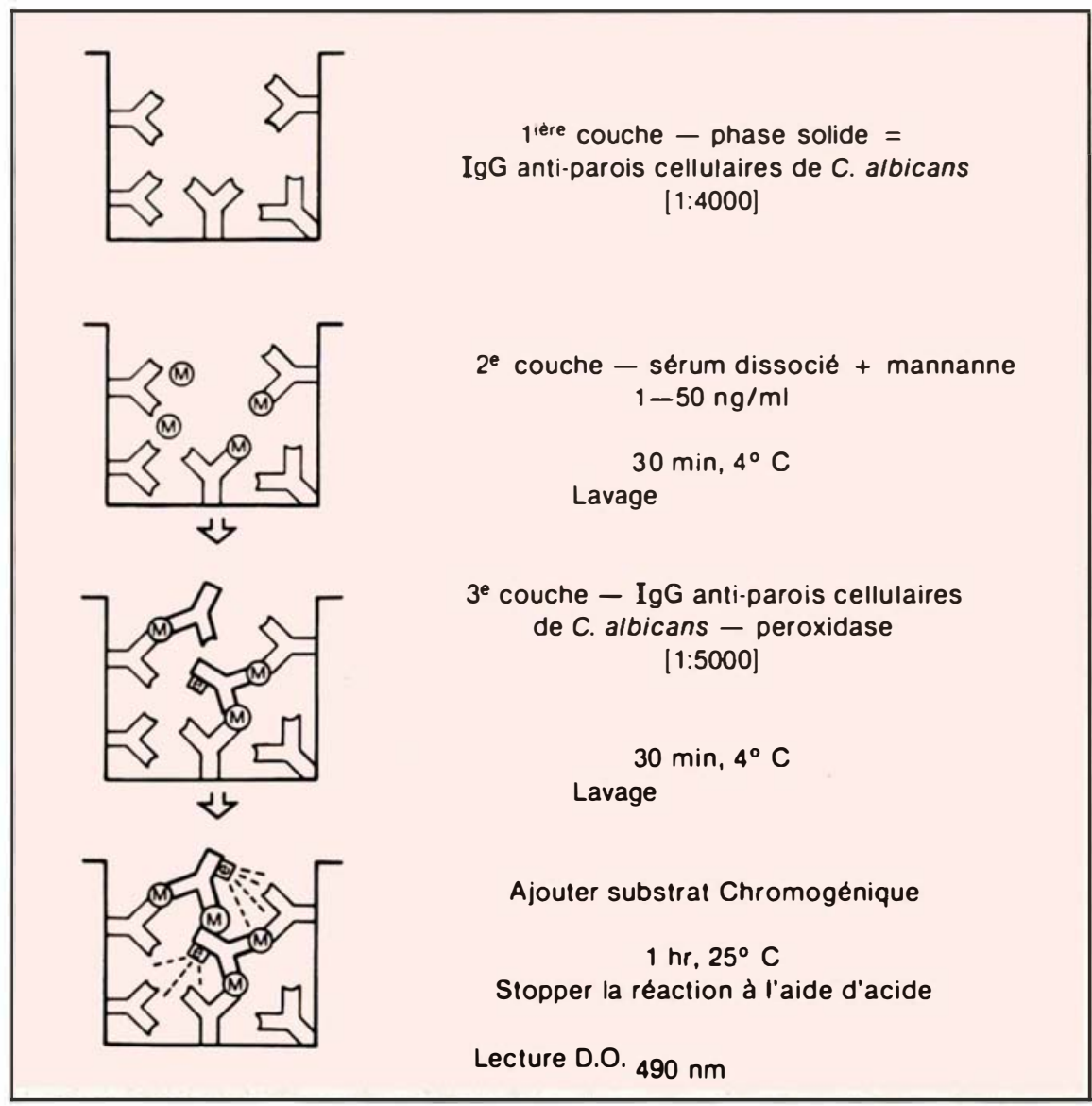

Figure 2. Réallsatlon de la détectlon de mannanne par méthode immunoonzymatlque de type sandwich. 


\section{RÉFÉRENCES}

16. Axelsen NH, Kirkpatrick CH. Simultaneous characterization of free Candida antigens and Candida precipitins in a patient's serum by means of crossed immunoelectrophoresis with intermediate gel. $J$ Immunol Methods 1973 ; 2 : 245-9.

17. De Repentigny L, Kuykendall RJ, Chandler FW, Broderson JR, Reiss E. Comparison of serum mannan, arabinitol, and mannose in experimental disseminated candidiasis. $\mathrm{J}$ Clin Microbiol 1984 ; 19 : 804-12.

18. De Repentigny L, Marr LD, Keller JW, et al. Comparison of enzyme immunoassay and gas-liquid chromatography for the rapid diagnosis of invasive candidiasis in cancer patients. J Clin Microbiol 1985 ; 21 : 972-9.

19. Reiss E, de Repentigny L, Kuykendall $\mathrm{RJ}$, et al. Monoclonal antibodies against Candida tropicalis mannan : antigen detection by enzyme immunoassay and immunofluorescence. J Clin Microbiol 1986 ; 24 : 796-802.

20. Araj GF, Hopfer RL, Chesnut S, Fainstein V, Bodey GP. Diagnostic value of the enzyme-linked immunosorbent assay for detection of Candida albicans cytoplasmic antigen in sera of cancer patients. J Clin Microbiol 1982 ; $16: 46-52$.

21. Bailey JW, Sada E, Brass C, Bennett JE. Diagnosis of systemic candidiasis by latex agglutination for serum antigen. $J$ Clin Microbiol 1985 ; 21 : 749-52.

22. De Repentigny L, Kuykendall RJ, Reiss E. Simultaneous determination of arabinitol and mannose by gas-liquid chromatography in experimental candidiasis. $J$ Clin Microbiol 1983 ; $17: 1166-9$

23. Reiss E, Lehmann PF. Galactomannan antigenemia in invasive aspergillosis. Infect Immun 1979 ; 25 : 357-65.

24. Azuma I, Kimura $H$, Hirao F, Tsubura E, Yamamura Y. Biochemical and immunological studies on Aspagillus. I. Chemical and biological investigation of lipopolysaccharide, protein and polysaccharide fractions isolated from Aspagillus fumigatus. Jpn J Med Mycol $1967 ; 8: 210-20$.

25. Suzuki M, Hayashi $Y$. Skin reaction and macrophage migration inhibition tests for polysaccharides from Aspargillus fumigatus and Candida albicans. Jpn J Microbiol 1975; 19 : 355-62.

26. Azuma I, Kimura H, Hirao F, Tsubura E, Yamamura Y, Misaki A. Biochemical and immunological studies on Aspergillus. III. Chemical and immunological properties of glycopeptide obtained from Aspagillus fumigatus. Jpn $J$ Microbiol 1971 ; 15 : 237-46.

27. Bardalaye PC, Nordin JH. Chemical structure of the galactomannan from the cell wall of Aspergillus niger. J Biol Chem $1977 ; 252$ : 2584-91.

28. Drutz DJ. Antigen detection in fungal
Dans une étude rétrospective faite chez des cancéreux atteints de candidose invasive, le rapport arabinitol/créatinine avait une sensibilité de $13 \%$ et une spécificité de $93 \%$ [18].

On voit donc que la chromatographie en phase gazeuse et les techniques immunoenzymatiques ont chacunes des avantages et des inconvénients. La chromatographie en phase gazeuse permet de détecter directement les métabolites circulants sans avoir à préparer un antisérum et à le conjuguer à la peroxydase. Les méthodes immunoenzymatiques ont l'avantage de la spécificité immunologique et permettent de doser de nombreux sérums en trois ou quatre heures.

\section{Aspergillus}

Le diagnostic immunologique de l'aspergillose invasive chez l'hôte immunodéprimé a été gêné par la faible sensibilité et le peu de spécificité des techniques de dosage des anticorps [7]. Une approche différente a donc été envisagée, basée sur la détection de l'antigène galactomannanne.

Le galactomannanne des espèces Aspergillus est situé dans la paroi cellulaire de même que d'autres polysaccharides tels le glucane et le galactane. Il peut être isolé à partir du mycélium par extraction alcaline à froid [23] ou par extraction à la pyridine [24] suivie d'une précipitation à l'acétone [24] ou également à partir du filtrat de culture par précipitation au méthanol [25]. La structure du galactomannanne a été élucidée par différentes techniques : chromatographie en phase gazeuse, dégradation de Smith, acétolyse ménagée, étude de la liaison à la concanavaline $\mathrm{A}$ et hydrolyse en milieu acide. Le galactomannanne d'A. fumigatus $[23,26]$ contient une chaîne principale de mannanne liée par des liaisons $\alpha(1,2)$ ou $\alpha(1,6)$ et des chaînes latérales de galactose de trois unités chacune terminées par un galactofuranose. On suspecte également la présence de chaînes latérales de D-mannose liées $(1,6)$. Le galactomannanne d'A. niger est différent de celui d'A. fumigatus : il ne possède pas de chaînes latérales de mannose et les chaînes latérales de galactose peuvent comporter trois ou quatre unités [27]. Ces deux galactomannannes se lient à la concanavaline A. Des analyses plus poussées, telles que l'identification des monosaccharides méthylés par spectrométrie de masse et l'utilisation de la résonance magnétique nucléaire, devraient permettre de concilier les différentes interprétations de la structure du galactomannanne d'A. fumigatus. On pourra ainsi non seulement déterminer les liaisons internes de la chaîne principale oligomannosidique et celles des chaînes latérales oligogalactosidiques, mais encore confirmer la présence et les liaisons internes des chaînes latérales oligomannosidiques (figure 3). De plus, l'acétolyse ménagée permettrait d'isoler des fragments de chaînes latérales pouvant être utilisés dans des études d'inhibition par les haptènes à l'aide d'anticorps monoclonaux antigalactomannanne. Ces expériences permettraient de définir plus précisément les épitopes immunodominants du galactomannanne.

Reiss et Lehmann [23] ont rapporté les premiers la présence de galactomannanne dans le sérum de patients atteints d'aspergillose invasive. En injectant le sérum antigénémique d'un lapin infecté expérimentalement avec $A$. fumigatus à un autre lapin, ils ont obtenu un antisérum capable de reconnaître un antigène circulant (en contre-immuno-électrophorèse). Cet antigène circulant $a$ un poids moléculaire supérieur à 125000 , tandis que l'antigène purifié par extraction alcaline du mycélium a un poids moléculaire situé entre 25000 et 75000 . Cette différence peut être due à une liaison du galactomannanne à des protéines plasmatiques [23]. Enfin, un test radioimmunologique de type compétitif a été récemment mis au point. Il permet de détecter un antigène qui est un hydrate de carbone et qui pourrait être le galactoman$m / s \quad n \circ 2$ ool. 3, ftorier 87 


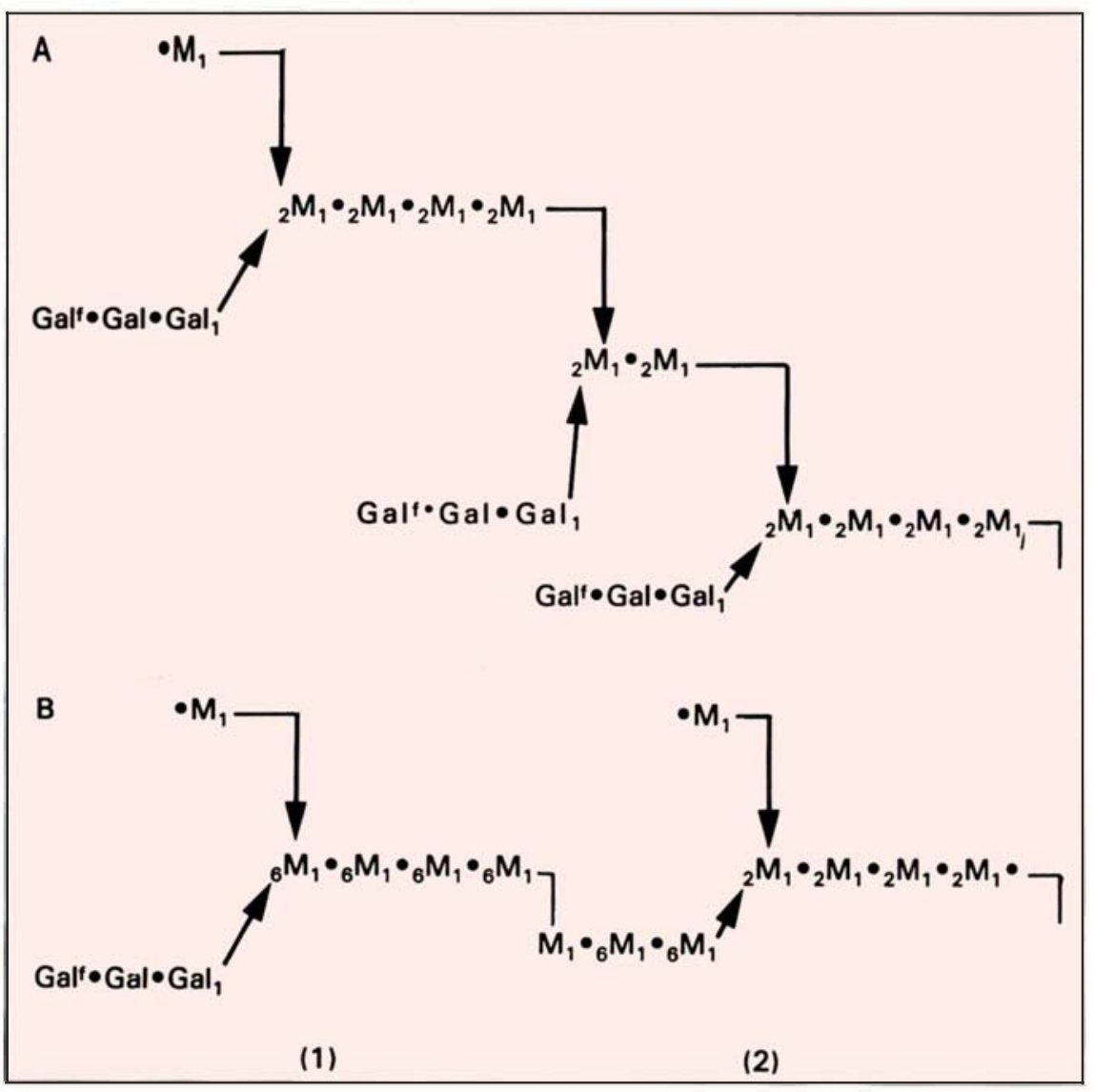

Figure 3. (A) Modèle de la structure du galactomannanne d'Aspergillus fumigatus. Le polysaccharide contient une chaîne principale de mannanne liée $\alpha(1,2)$, et des chaines latérales de galactose de trois unités chacune et terminées par un galactofuranose. (B) On retrouve également des oligomannosides liés $(1,6)$ qui font partie de la chaîne principale (1) ou qui forment de courtes chaînes latérales (2).

nanne [7]. Cependant, ce test est relativement peu sensible $(100 \mathrm{ng} / \mathrm{ml}$ de sérum) comparé au test immunoenzymatique de type sandwich. De plus, les réactifs ont une demi-vie assez courte et posent le problème des déchets radioactifs. Cette méthode a néanmoins permis de détecter l'antigène dans le sérum, le liquide pleural et le liquide de lavage bronchoalvéolaire provenant de patients atteints d'aspergillose invasive. La sensibilité de la méthode radioimmunologique est de $75 \%$ et sa spécificité est de presque $100 \%$.

En conclusion, la détection d'antigènes circulants au cours des maladies fongiques off re de nouvelles possibilités de diagnostic rapide, en particulier chez l'hôte immunodéprimé [28]. Les polysaccharides de la parol cellulaire de Candida et d'Aspergillus circulent dans le sérum de patients atteints de candidose et d'aspergillose invasives et peuvent être rapidement détectés par des méthodes immunoenzymatiques. Il est possible, grâce à ces méthodes, d'envisager un diagnostic et un traitement précoces permettant d'améliorer le pronostic chez les patients atteints de mycoses opportunistes. Cependant, les techniques diagnostiques dont nous disposons actuellement devraient être améliorées avant d'être largement appliquées en clinique. Nous travaillons actuellement à la mise au point d'un test immunoenzymatique de type sandwich utilisant un anticorps monoclonal anti-galactomannanne

\section{Summary}

Invasive candidiasis and aspergillosis are a growing problem in the immunocompromised host. Approaches to prevention of these fungal infections have had limited success. Cultures of superficial sites and of blood do not provide the required diagnostic accuracy and serological tests that detect antibody to the Candida and Aspergillus species have an insufficient predictive value in immunosuppressed patients. Detection of the cell wall polysaccharide antigens marinan of Candida and galactomannan of Aspergillus appears promising for the rapid diagnosis of these lifethreatening fungal infections.

\section{Remerciements}

Les auteurs remercient le docteur Errol Reiss (Division of Mycotic Diseases, Centers for Disease Control, Atlanta, Georgia) pour sa précieuse collaboration aux études résumées dans cet article.

\section{TIRÉS A PART}

L. de Repentigny : département de microbiologie et d'immunologie, faculté de médecine, université de Montréal, C.P. 6128, Succ. A, Montréal, Québec, Canada H3C 3J7. 